\title{
Usefulness of new live 3D ultrasound probe during radiofrequency ablation in a case of hepatocellular carcinoma
}

\author{
Naoki Hotta*, Kazuo Masuko \\ Department of Internal Medicine, Division of Hepatology, Masuko Memorial Hospital, Aichi, Japan \\ Email: ${ }^{*}$ naokiki@aol.com
}

Received 31 December 2011; revised 1 February 2012; accepted 13 February 2012

\begin{abstract}
We report a case of hepatocellular carcinoma (HCC) with chronic hepatitis C virus (HCV) infection, successfully treated with percutaneous radiofrequency ablation (RFA) under new Live trhree-dimensional (3D) echo guidance for hearts probe before [1], but ,at the time, perform RFA with a new Live3D probe developed flants. A 61 year-old Japanese man had a 3.5 $\mathrm{cm}$ in diameter HCC nodule in the liver S3 region. We applied the Live 3D ultrasonography during RFA therapy with a cool-tip needle electrode. The echo guidance facilitated an accurate approach for the needle puncture. The guidance was also useful for confirming, whether an adequate safety marging for the nodule had been obtained. Thus new Live 3D ultrasonography echo technique appears to provide safe guidance of RFA needles via accurate targeting of HCC nodules, thereby allowing real-time visualizetion when combined with echo contrast.
\end{abstract}

Keywords: New Live; 3D Probe; HCC

\section{INTRODUCTION}

Hepatocellular carcinoma (HCC) is increasing worldwide including in the United States and Europe, and is thus a global problem [2,3]. Patients with chronic liver disease related to hepatitis $\mathrm{B}$ or $\mathrm{C}$ virus constitute a high risk group for the development of HCC [4]. Surgical resection for HCC has a limited role because of underlying cirrhosis or the presence of multiple HCC lesions. Accordingly various non-surgical therapies have been attempted. Among them, percutaneous therapies such as percutaneous ethanol injection (PEI), microwave coagulation and radiofrequency ablation (RFA) have been widely applied [5]. RFA may be the most promising of these treatment strategies. Studies evaluating the tumor vascularity of HCC have been conducted extensively

${ }^{*}$ Corresponding author. using various imaging modalities.

The recent advances in ultrasonography (US) devices in conjunction with better contrast agents have greatly improved sensitivity in evaluating the hemodynamics of liver tumors based on the availability of real-time grayscale harmonic US imaging [6]. Since we are expected to ablate the tumor nodule with an adequate safety margin, a more accurate procedure and positioning of the inserted needle during RFA employing more advanced devices is clearly desirable. Herein we report a case with HCC successfully treated with percutaneous RFA under the new Live 3D ultrasonography guidance.

\section{CASE REPORT}

A 61year-old Japanese man was referred to our outpatient clinic in December 2011 for treatment of a hepatic tumor. He had no remarkable past medical history other than chronic hepatitis $\mathrm{C}$ which had been followed at another outpatient clinic since 2005 A hepatic tumor on abdominal $\mathrm{CT}$ was detected at a periodic examination in November 2011. He was admitted to our gastrointestinal (GI) ward in December 2011. On admission, his physical examination findings were unremarkable; neither the liver nor the spleen was palpable. There was no jaundice, ascites, peripheral edema or other signs of chronic liver disease. Laboratory tests results included a white blood cell count of $3910 / \mu \mathrm{L}$, hemoglobin $13.8 \mathrm{~g} / \mathrm{dL}$, and a platelet count of $65,000 / \mu \mathrm{L}$. Liver biochemistry tests showed increased serum transaminases levels (aspartate aminotransferase $79 \mathrm{IU} / \mathrm{L}$ and alanine aminotransferase $70 \mathrm{IU} / \mathrm{L})$. Other laboratory tests results were within normal ranges.The $\alpha$-fetoprotein (AFP) was levels $35 \mathrm{mAU} /$ $\mathrm{mL}$ and low vitamin $\mathrm{K}$ absence-2 (PIVKA-2) was 35 $\mathrm{mAU} / \mathrm{ml}$ (Table 1). He was seropositive for anti-HCV by a second-generation enzyme immunoassay (Dainabot, Tokyo, Japan) The fundamental B-mode US scan showed the presence of a low echoic $3.5 \mathrm{~m}$ in diameter lesion in the $\mathrm{S} 3$ area. On dynamic CT, the nodule showed tumor 
staining in the early arterial phase and wash-out density in the post phase.

We applied as contrast-enhanced US with a X6-1 probe from IU22 (Phillips Electronics Japan.Ltd.Medical Systems). The vascularity of the HCC lesion was assessed by Contrast-enhanced 4D imaging using Sonazoid (Daiichi-Sankyo Tokyo, Japan) $0.5 \mathrm{ml}$ of Sonazoid [7].

The US imaging was focused at the base of the target tumor. The images were taken in the early vascular phase from $10 \mathrm{sec}$ until $40 \mathrm{sec}$ after injection; tumor vessel and staining were clearly observed (Figure 1). The CT and US findings indicated that the patient had HCC. Since he refused angiography or surgery, RFA was selected as the therapy.

RFA was conducted for 12 min under a percutaneous approach using a cool-tip needle (COVIDIEN) with a 3.0 $\mathrm{cm}$ in diameter hook. During and after the procedure, Live 3D ultrasonography was applied for the accurate positioning of the needle and for confirmation of adequate ablation with a sufficient safety margin (Figure 2). We

Table1. Laboratory data on admission.

\begin{tabular}{lll}
\hline Chemistry & CBC & Coagulation \\
\hline TP: $7.0 \mathrm{~g} / \mathrm{dl}$ & WBC: $3910 / \mu \mathrm{l}$ & APTT: $35.5 \mathrm{~s}$ \\
Alb: $3.3 \mathrm{~g} / \mathrm{dl}$ & RBC: $406 \times 10^{4} / \mu \mathrm{l}$ & PT-T: $13.3 \mathrm{~s}$ \\
T-Bil: $0.6 \mathrm{mg} / \mathrm{dl}$ & Hgb: $13.8 \mathrm{~g} / \mathrm{dl}$ & PT\%: $77 \%$ \\
AST: $79 \mathrm{IU} / 1$ & Hct: $39.7 \%$ & PT-INR: 1.15 \\
ALT: $78 \mathrm{IU} / 1$ & MCV: $97.9 \mathrm{fl}$ & \\
ALP: $201 \mathrm{IU} / 1$ & Plt: $6.5 \times 10^{4} / \mu 1$ & (Tumor Marker) \\
$\gamma$-GTP: $80 \mathrm{IU} / 1$ & & AFP: $34.1 \mathrm{ng} / \mathrm{ml}$ \\
LDH: $201 \mathrm{IU} / 1$ & & $\underline{\text { PIVKAII: } 35}$ \\
Na: $139 \mathrm{mEq} / 1$ & & \\
K: $4.1 \mathrm{mEq} / 1$ & & \\
Cl: $105 \mathrm{mEq} / 1$ & & $\underline{\text { HCV-Ab positive }}$ \\
BUN: $8 \mathrm{mg} / \mathrm{dl}$ & & $\underline{\text { HBs0Ab negative }}$ \\
Cre: $0.52 \mathrm{mg} / \mathrm{dl}$ & & \\
\hline
\end{tabular}

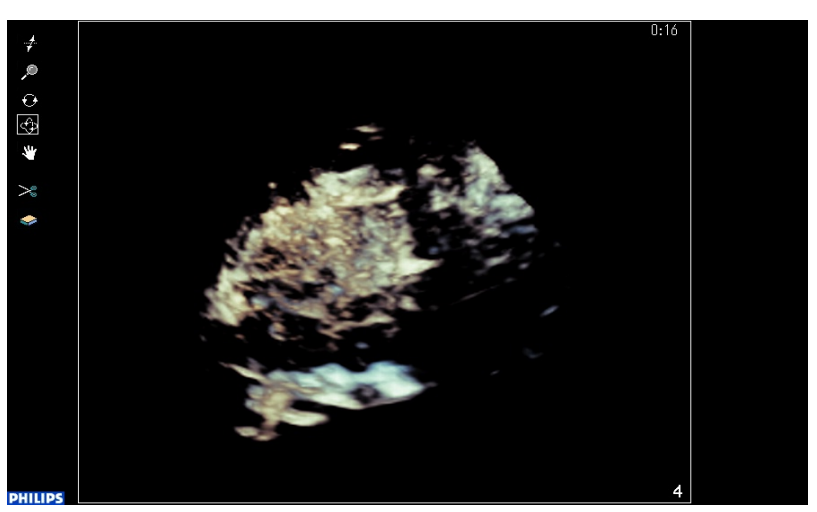

Figure 1. On contrast-enhanced US, $3.5 \mathrm{~cm}$ in diameter tumor stainig can be seen in the liver S3 area in the early arterial phase. also confirmed the position of the needle using the multiview detector with which this model is equipped, and the solid mode which allows depiction of lesions in units of several $\mathrm{mm}$ as one area as on a CTscan. Dynamic CT scan performed 1 day after RFA showed no tumor staining with a low density area corresponding to the tumoraffected part of the liver in the early arterial phase. Dynamic CT also indicated an adequate safety margin for the nodule.

After RFA tretment, contrast-enhanced ultrasonography was performed and the site of the enhanced tumor was visualized as a defect (Figure 3).

\section{DISCUSSION}

Three-dimensional US (3DUS) and four-dimensional US (4DUS) contrast enhanced US (CEUS) include US, and there is many examination with the probe of mechanical,

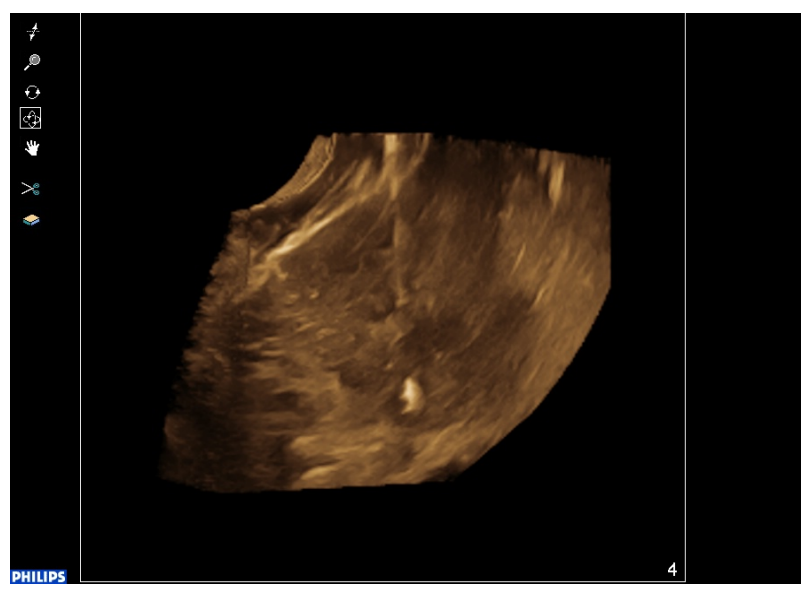

Figure 2. On live 3D mode in US, needle puncture during the RFA procedure and insertion into the center of tumor nodule were confirmed. We also confirmed expansion of the cool-tip needle from the under portion of the tumor.

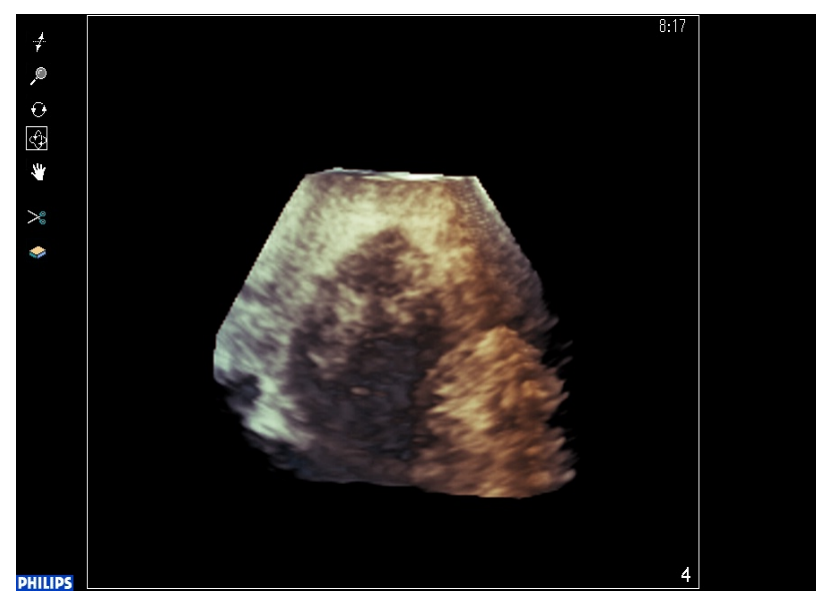

Figure 3. After treatment,contrast-enhanced ultrasonography was performed and the site of the enhanced tumor was visualized as a defect. 
but, at the examination with the matrix array probe,this is the first report.

US is the most commonly used imaging method for diagnosing hepatic tumors. The improvement of US devices with the contrast agents appears to contribute to increased sensitivity and accuracy in evaluating the hemodynamics of hepatic tumors e.g. the development of new method with 3D imaging. This study demonstrated the possibility, if easily assessing intra-tumoral blood flow dynamics in patients with hepatic tumors [7,8]. In addition, with the use of a contrast agent, Sonazoid, small vessels and low perfusion could be depicted in real-time. The probe which we used herein was a microconvex type probe which is smaller than that we reported previously [1]. For the trace, we observed the needle and anticipate its future use for guiding accurate puncture. Despite improved US devices, we occasionally experience cases in which accurate positioning of the RFA needle is difficult because the nodules are indistinct. There are disadvantages to using 4D US e.g. that current 4D ultrasonic transducers are bulkier and heavier than conventional 2D transducers, resulting in some difficulty in handling the probe. Moreover, the mechanical sweeping motion of the 4D transducer generated a mechanical vibration during the RFA procedure.

We used a new Live 3D ultrasonography device for RFA therapy in the present study and confirmed that the needle was inserted accurately into the center of the target tumor. The tumor was ablated with an adequate safety margin. At the time of puncture matrix array probe, there was a fauit to show the vibration of the probe, but, as for this probe, treatment was enabled without the frame rate decreasing without vibration. We easily and quickly obtained useful information regarding positioning of the needle. Since this device can depict the tumor in all sections, it is possible to confirm the three-dimensional positional relationships among the target, needle and adjacent organs such as large blood vessels, the gall bladder and the diaphragm. This will allow the RFA procedure to be performed more safely.

The technique described herein using a new Live 3D US device has the limitation that the probe lacks guidance for the puncture. Thus it can serve as a diagnostic but not as a therapeutic tool. It would be optimal to have the puncture guidance along with development of the corresponding contrast agents.

If realtime $3 \mathrm{D}$ puncture is enable by the device which we can weigh the CT image, as for the future examination, it seems that the therapeutic safety improves than naw.

\section{REFERENCES}

[1] Hotta, N., Ayada, M., Okumura, A., Ishikawa, T. and Kakumu, S. (2007) Usefulness of live 3D echocardiography during radiofrequency ablation in a case of hepatocellular carcinoma. Clinical Imaging, 31, 283-286.

doi:10.1016/j.clinimag.2007.01.009

[2] El-Serag, H.B. and Mason, A.C. (1999) Rising incidence of hepatocellular carcinoma in the United States. The New England Journal of Medicine, 340, 745-750. doi:10.1056/NEJM199903113401001

[3] Taylor-Robinson, S.D., Foster, G.R., Arora, S., Hargreaves, S. and Thomas, H.C. (1997) Increase in primary liver cancer in the UK 1979-94. Lancet, 350, 1142-1143. doi:10.1016/S0140-6736(05)63789-0

[4] Shiratori, Y., Shiina, S., Imamura, M., Kato, N., Knai, F., Okudaira T., Teratani, T., Tohgo, G., Toda, N. and Omata, M. (1995) Characteristic difference of hepatocelluar carcinoma between hepatitis B- and C- viral infection in Japan. Hepatology, 22, 1027-1033. doi:10.1002/hep.1840220403

[5] Livraghi, T., Lazzaroni, S. and Meloni, F. (2001) Radiofrequency thermal ablation of hepatocellular carcinoma. European Journal of Ultarasound, 13, 159-166. doi:10.1016/S0929-8266(01)00128-8

[6] Hotta, N., Maeno, T., Ayada, M., Sato, K., Ishikawa, T., Okumura, A., Matsumoto, E., Fukuzawa, Y. and Kakumu, S. (2006) Four-dimensional ultrasonography for therapeutic radiofrequency ablation for hepatocellular carcinoma. Hepato-Gastroenterology, 53, 521-525.

[7] Wen, L., Numata, K., Morimoto, M., Kondo, M., Takebayashi, S., Morita, S. and Tanaka, K. (2009) Focal Liver Tumors: Characterization with 3D perflubutane microbubble contrast agent; enhanced US versus 3D contrastenhanced multidetector CT. Radiology, 251, 287-295. doi:10.1148/radiol.2511081324

[8] Numata, K., Luo, W., Morimoto, M., Kondom M., Kunishi, Y., Sasaki, T., Nozaki, A. and Tanaka, K. (2010) Contrast enhanced ultrasound of hepatocellular carcinoma. World Journal of Radiology, 28, 68-82. doi:10.4329/wjr.v2.i2.68 